\title{
Performance Improvement of Sparse Matrix Vector Product on Vector Machines
}

\author{
Sunil R. Tiyyagura ${ }^{1}$, Uwe Küster ${ }^{1}$, and Stefan Borowski ${ }^{2}$ \\ ${ }^{1}$ High Performance Computing Center Stuttgart, \\ Allmandring 30, 70550 Stuttgart, Germany \\ \{sunil, kuester\}@hlrs.de \\ 2 NEC HPCE GmbH, Heßbrühlstr. 21B, 70565 Stuttgart, Germany \\ sborowski@hpce.nec.com
}

\begin{abstract}
Many applications based on finite element and finite difference methods include the solution of large sparse linear systems using preconditioned iterative methods. Matrix vector multiplication is one of the key operations that has a significant impact on the performance of any iterative solver. In this paper, recent developments in sparse storage formats on vector machines are reviewed. Then, several improvements to memory access in the sparse matrix vector product are suggested. Particularly, algorithms based on dense blocks are discussed and reasons for their superior performance are explained. Finally, the performance gain by the presented modifications is demonstrated.
\end{abstract}

Keywords: Matrix vector product, Jagged diagonal storage, Vector processors.

\section{Introduction}

The main challenge facing computational scientists and engineers today is the rapidly increasing gap between sustained and peak performance of the high performance computing architectures. Even after spending considerable time on tuning applications to a particular architecture, this gap is an ever existing problem. Vector architecture provides a reasonable solution (at least up to certain extent) to bridge this gap [1. The success of the Earth Simulator project 2] also emphasizes the need to look towards vector computing as a future alternative.

Sparse iterative solvers play an important role in computational science to solve linear systems arising from discretizing partial differential equations in finite element and finite difference methods. The major time consuming portions of a sparse iterative solver are the matrix vector product (MVP) and preconditioners based on domain decomposition like ICC, ILU, BILU, etc. The MVP algorithm depends on the storage format used to store the matrix non-zeros. Storage formats can be broadly classified as row, column or pseudo diagonal oriented formats. Commonly used examples for each of the formats are compressed row storage (CRS), compressed column storage (CCS) and jagged diagonal storage (JAD). A detailed discussion of different storage formats along with 
corresponding conversions can be found in [3]. As row/column formats store the matrix non-zero entries of each row/column, the MVP algorithm in these cases is restricted to work on a single row/column in the innermost loop. This hinders the performance on vector machines if the number of non-zeros per row/column is less than the length of hardware vector pipeline. In case of NEC SX-8, a classical vector machine, the pipelines are 256 words long. Therefore, at least 256 non-zero entries per row/column are needed to have optimal performance on this architecture. If start-up time (pipeline depth and starting latencies) is additionally considered, the required number of entries for optimal performance is even larger.

Pseudo diagonal formats like the JAD storage are commonly used on vector machines as they fill up the vector pipelines and result in long average vector length. The length for the first few pseudo diagonals is equal to the size of the matrix and then decreases for the latter depending on the kind of problem. This helps in filling up the vector pipelines which results in superior performance over other formats for MVP. However, pseudo diagonal formats are not as natural as the row/column formats which makes their initial setup difficult. In the paper on hand, we focus on performance issues of the sparse MVP algorithm on vector machines. Different approaches to optimize the memory access pattern in this algorithm are addressed. The reduction of load/store operations for the result vector is regarded by modifying the algorithm and using vector registers. The more challenging problem of reducing the indirect memory access for the multiplied vector is considered by introducing a block based algorithm.

In Section 2 of the paper, we take a closer look at some recently proposed improvements for sparse storage formats. In Section 3, the changes decreasing the memory access in the result vector are explained. Then, the block based MVP algorithm is discussed. In Section 4, performance results are presented for the proposed changes. Section 5 summarizes the outcome of this paper.

\section{Recent studies}

There have been recent studies on optimizing sparse storage formats. It is worth understanding the implications of this work in the context of typical vector processing.

\subsection{Optimizing the Storage}

Transposed jagged diagonal format (TJAD) optimizes the amount of storage needed for a sparse matrix by eliminating the need for storing the permutation vector [4. However, this forces a shift of indirect addressing from the multiplied vector to the result vector. As the result vector has to be both loaded and stored, this effectively doubles the amount of indirect addressing needed for MVP. This is a matter of concern on conventional vector architecture, but not much on cache based machines. Since this format is principally used on vector machines, this cannot be the desired alternative. 


\subsection{Improving Average Diagonal Length}

Bi-jagged diagonal storage (Bi-JDS) combines both common JAD and TJAD to further increase the average length of diagonals $[5$. The main idea is to store all the full length diagonals in JAD format and the remaining matrix data in TJAD format. TJAD has the disadvantage of increasing the indirect addressing as explained in the previous section. Setting up such a format within the scope of the whole iterative solver would cause changes to all algorithms that use the matrix data such as preconditioning. Furthermore, a lot of problems yield matrices with satisfactorily long average vector lengths in JAD format like matrices from structured finite element, finite difference and finite volume analysis. For problems involving surface integrals, it is common that the number of non-zeros in only some of the rows are extremely high. In this case, it may be advantageous to use such a scheme. This has to be extensively tested to measure its pros (long diagonals) against its cons (set-up cost and doubled indirect addressing).

\subsection{Row Format on Vector Machines}

Compressed row storage with permutation (CRSP) was introduced in [6]. Results for this format on Cray X1 show a performance of the MVP algorithm that is an order of magnitude higher than for common CRS format. The permutation introduced in the CRSP format adds a level of indirection to both the matrix and the multiplied vector, i.e. effectively two additional indirectly addressed memory loads per loop iteration. This is the overhead incurred because of permuting the rows in ascending order of their length. Although this results in tremendous performance improvement on Cray X1 due to caching, such an algorithm would perform poorly on conventional vector machines because of the heavy cost of indirect addressing involved (in absence of cache memory). The overhead of indirection in memory access is elaborated in the next section.

\section{Improvements to the Algorithm}

Here, several changes to the JAD MVP algorithm are proposed, which improve the performance on vector machines. In order to reduce memory access for the result vector, an algorithmic approach is to operate on more than one diagonal in the innermost loop. A technical alternative is the use of vector registers offered by vector machines. Finally, the more critical issue of reducing indirect memory access for the multiplied vector is addressed by considering block based algorithms. Before introducing the changes, a brief introduction to features of a vector architecture is provided.

\subsection{Vector Processor}

Vector processors like NEC SX-8 use a very different architectural approach than scalar processors. Vectorization exploits regularities in the computational structure to accelerate uniform operations on independent data sets. Vector arithmetic 
instructions are composed of identical operations on elements of vector operands located in vector registers.

For non-vectorizable instructions the NEC SX-8 processor also contains a cache-based superscalar unit. Since the vector unit is by far more powerful than the scalar unit, it is crucial to achieve high vector operation ratios, either via compiler discovery or explicitly through code and data (re-)organization. The vector unit has a clock frequency of $2 \mathrm{GHz}$ and provides a peak vector performance of $16 \mathrm{GFlop} / \mathrm{s}$ ( 4 add and 4 multiply pipes working at $2 \mathrm{GHz}$ ). The total peak performance of the processor is $22 \mathrm{GFlop} / \mathrm{s}$ (including divide/sqrt unit and scalar unit). Table 1 gives an overview of the different processor units.

Table 1. NEC SX-8 processor units

\begin{tabular}{|l|c|c|}
\hline Unit & $\begin{array}{c}\text { No. of results } \\
\text { per cycle }\end{array}$ & Peak (GFlop/s) \\
\hline Add & 4 & 8 \\
Multiply & 4 & 8 \\
Divide/sqrt & 2 & 4 \\
Scalar & & 2 \\
\hline & \multicolumn{2}{|c|}{} \\
\hline
\end{tabular}

\subsection{Original JAD MVP Algorithm}

The original JAD MVP algorithm is listed in Fig. 1. For simplicity, the permutation of the result vector is not shown. The performance limitations of this algorithm can be better understood in terms of an operation flow diagram shown in Fig. 2, which explains the execution of the vector instructions. It should be noted that there is only one load/store unit in the NEC SX-8 processor. In each clock cycle, two floating point (FP) operations per pipeline are possible : 1 add and 1 multiply. The main bottleneck of this algorithm is the indirect load of the multiplied vector (vec) which takes roughly 5 times longer than a directly addressed one on NEC SX-8. On superscalar architectures, this factor is in general even greater and more complicated to predict.

In 9 cycles ( 5 for indirectly addressed vector), the possible number of FP operations is 18 (add and multiply). But the effective FP operations in the innermost loop of the algorithm are only 2 (1 add and 1 multiply). Hence, the expected performance of this operation would be $2 / 18$ th of the vector peak.

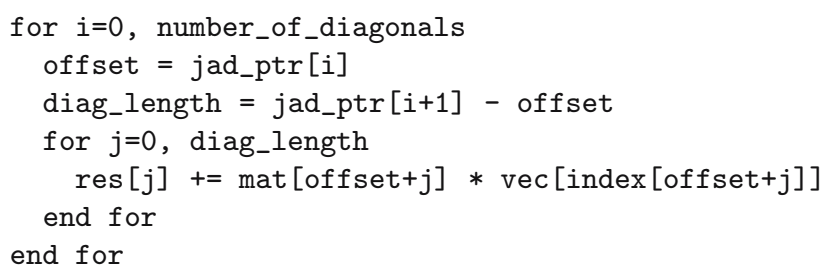

Fig. 1. Original JAD MVP algorithm 


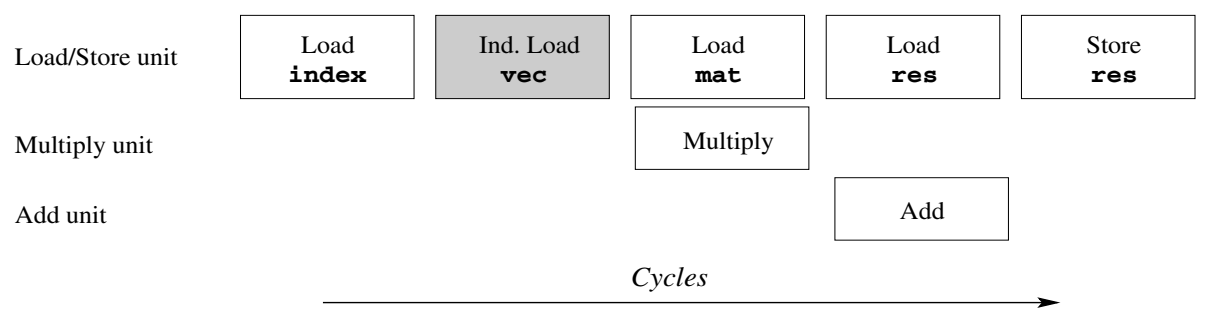

Fig. 2. Operation flow diagram for original JAD MVP algorithm

\subsection{Working on Groups of Diagonals}

Matrices originating from structured grids have groups of pseudo diagonals with equal length (stored in JAD format). One way to improve the performance is to operate on groups of equal length diagonals in the innermost loop instead of a single diagonal. This considerably saves load/store operations for the result vector (res). The accordingly modified algorithm is listed in Fig. 3. For simplicity, it works on utmost 2 diagonals of equal length. Extending this procedure to more diagonals improves the performance notably.

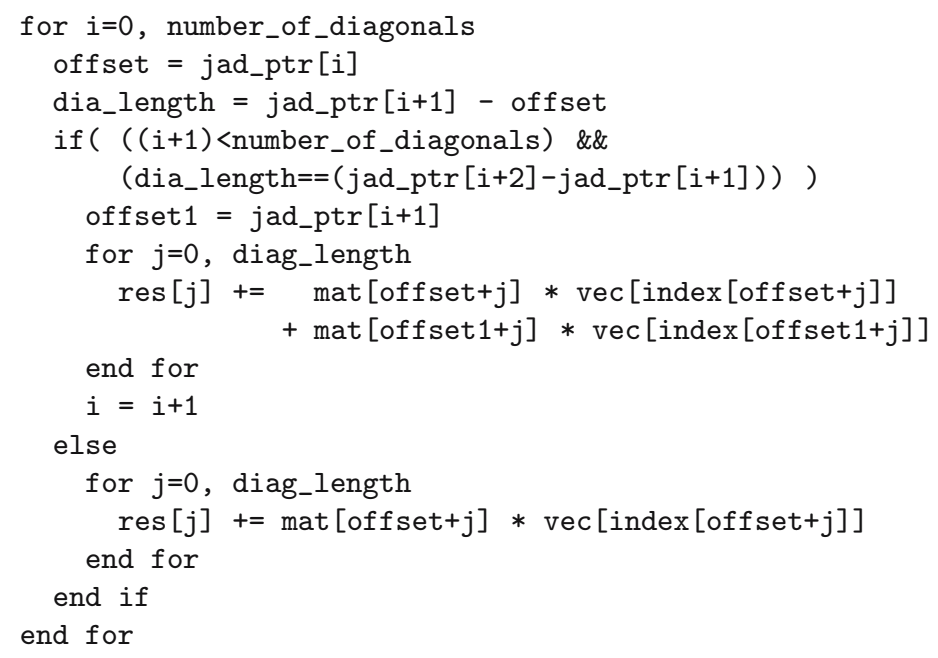

Fig. 3. JAD MVP algorithm grouping at most 2 diagonals

\subsection{Use of Vector Registers}

Most vector machines provide a programmer interface to vector registers in order to temporarily store data, like the result vector (res). Using vector registers would need the user to strip mine the innermost loop. The resulting algorithm is listed in Fig. 4. This procedure does not depend on the grid 


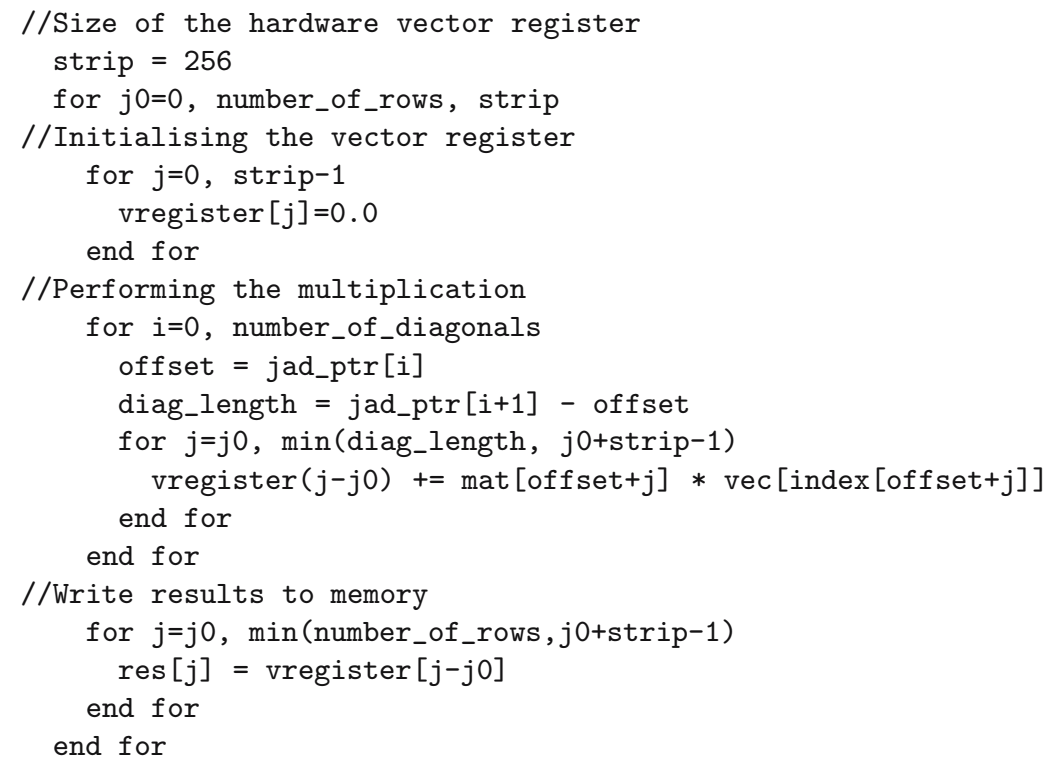

Fig. 4. JAD MVP algorithm using vector registers

(structured/unstructured) and hence equally reduces the memory access for the result vector.

\subsection{Operating on Dense Blocks}

In the point based algorithms discussed so far, the major hurdle to performance of MVP is indirect memory addressing. To overcome this, block based computations exploit the fact that many problems have multiple physical variables per node. Thus, small blocks can be formed by grouping the equations at each node. Operating on such dense blocks considerably reduces the amount of indirect addressing required for MVP. This improves the performance dramatically on vector machines [7] and also remarkably on superscalar architectures [8]. The block based algorithm for MVP is listed in Fig. 5] (block size 2 for simplicity). The reduction in indirect addressing can be clearly seen in the corresponding operation flow diagram shown in Fig. 6. The multiplied vector is indirectly addressed only twice (vec1 and vec2) instead of four times as it would be the case for the original algorithm. The needed index vector index is only loaded once and then incremented. To generalize, indirect memory addressing is reduced by a factor of block size ( 2 in this case).

\section{Performance Results}

The performance of the algorithm working on groups of diagonals is listed in Table 2. The case tested was a structured finite element problem with 8500 


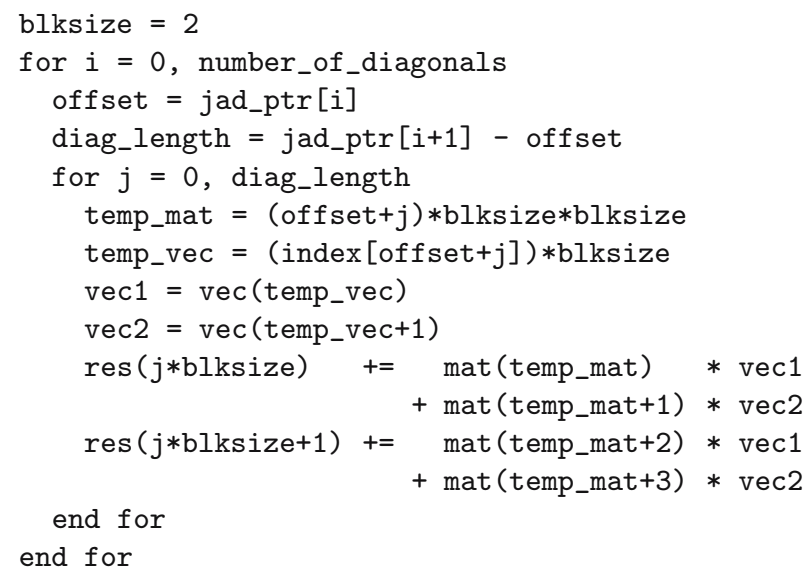

Fig. 5. Block based JAD MVP algorithm (for $2 \times 2$ blocks)

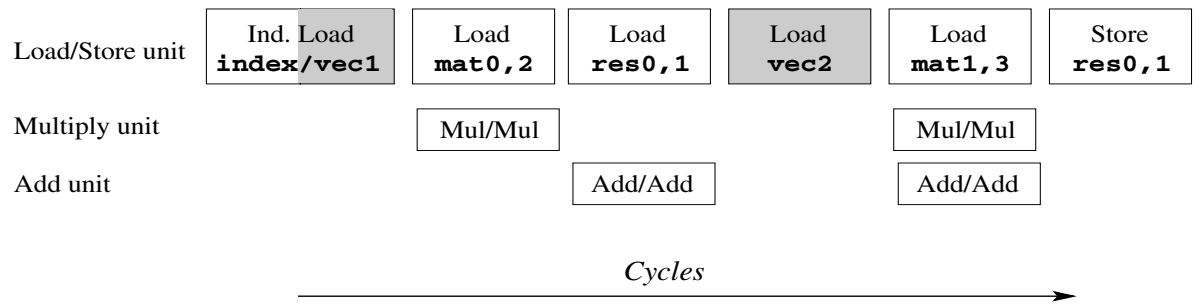

Fig. 6. Operation flow diagram for block based JAD MVP algorithm

hexahedral elements, 10065 nodes and 40260 unknowns. Increasing the group size improves the performance significantly. Grouping 9 diagonals results in a performance improvement of $78 \%$ over the original algorithm. Using vector registers, the performance of MVP was measured to be $2197 \mathrm{MFlop} / \mathrm{s}$, about $23 \%$ improvement compared to the original algorithm.

Table 2. Performance of JAD MVP algorithm grouping diagonals

\begin{tabular}{|c|c|}
\hline Max. group size & Performance (MFlop/s) \\
\hline orig & 1780 \\
3 & 2511 \\
5 & 2830 \\
9 & 3181 \\
\hline
\end{tabular}

Block based algorithms perform much better than the point based ones. For small test cases the performance of MVP for block sizes 4 and 5 is listed in Table 3. Operating on small dense blocks is a superior way to achieve a good 
percentage of the peak performance on vector machines ( $37 \%$ for block size 5$)$. The performance improvement over the original algorithm is $235 \%$. The improvement compared to the best performing point based algorithm introduced is $87 \%$.

Table 3. Performance of block based JAD MVP algorithm

\begin{tabular}{|c|c|}
\hline Block size & Performance (MFlop/s) \\
\hline orig & 1780 \\
4 & 5804 \\
5 & 5969 \\
\hline
\end{tabular}

\section{Summary}

Several improvements to the matrix vector product algorithm based on jagged diagonal storage for vector machines have been suggested in the paper. Most of the memory access problems in this key operation of sparse iterative solvers have been addressed. Block based algorithms are necessary to achieve a good portion of the peak performance on vector machines and moreover should also benifit superscalar architectures. Further work is still required to look for efficient preconditioning methods based on dense blocks.

\section{References}

1. Oliker, L., Canning, A., Carter, J., Shalf, J., Ethier, S.: Scientific computations on modern parallel vector systems. In: Proceedings of the ACM/IEEE Supercomputing Conference 2004, Pittsburgh, USA (2004)

2. http://www.es.jamstec.go.jp/esc/eng/.

3. Saad, Y.: SPARSKIT: A basic toolkit for sparse matrix computations. Technical Report RIACS-90-20, NASA Ames Research Center, Moffet Field, CA (1994)

4. Montagne, E., Ekambaram, A.: An optimal storage format for sparse matrices. Information Processing Letters 90 (2004) 87-92

5. Hossain, S.: On efficient storage of sparse matrices. In: 2005 Istanbul Computational Science and Engineering Conference (ICCSE '05), ITU, Istanbul (2005)

6. D'Azevedo, E.F., Fahey, M.R., Mills, R.T.: Vectorized sparse matrix multiply for compressed row storage format. In: Proceedings of the 5th International Conference on Computational Science, Atlanta, USA, Springer-Verlag (2005)

7. Nakajima, K.: Parallel iterative solvers of geofem with selective blocking preconditioning for nonlinear contact problems on the earth simulator. GeoFEM 2003-005, RIST/Tokyo (2003)

8. Tuminaro, R.S., Shadid, J.N., Hutchinson, S.A.: Parallel sparse matrix vector multiply software for matrices with data locality. Concurrency: Practice and Experience (3)10 (1998) 229-247 